\title{
Functional Connectivity in Mild Cognitive Impairment During a Memory Task: Implications for the Disconnection Hypothesis
}

\author{
Ricardo Bajo $0^{a, 1, *}$, Fernando Maestú ${ }^{a, b}, 1$, Angel Nevado ${ }^{a, b}$, Miguel Sancho , Ricardo Gutiérrez ${ }^{a}$, \\ Pablo Campo ${ }^{a}$, Nazareth P. Castellanos ${ }^{a}$, Pedro Gil ${ }^{\mathrm{d}}$, Stephan Moratti ${ }^{\mathrm{a}, \mathrm{e}}$, Ernesto Pereda ${ }^{\mathrm{f}}$ and \\ Francisco del-Pozo ${ }^{\mathrm{a}}$ \\ "Laboratory of Cognitive and Computational Neuroscience, Center of Biomedical Technology, Madrid. Spain

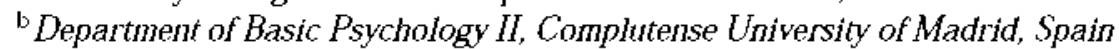 \\ ${ }^{c}$ Department of Applied Physics III of Complutense University of Madrid, Spain \\ d Department of Geriatrics (Memory Unit), San Carlos University Hospital. Madrid. Spain \\ 'Depastment of Basic Psychology I. Complutense University of Madrid. Spain \\ ${ }^{\mathbf{f}}$ Electrical Engineering and Bioengineering Group, Dept. of Basic Physics, University of La Laguna, Tenerffe, \\ Spain
}

\begin{abstract}
Mild cognitive impairment (MCI) has been considered an intermediate state between healthy aging and dementia. The early damage in anatomical connectivity and progressive loss of synapses that characterize early Alzheimer's disease suggest that MCI could also be a disconnection syndrome. Here. we compare the degree of synchronization of brain signals recorded with magnetoencephalography from patients (22) with MCI with that of healthy controls (19) during a memory task. Synchronization Likelihood, an index based on the theory of nonlinear dynamical systems. was used to measure functional connectivity. During the memory task patients showed higher interhemispheric synchronization than healthy controls between left and right -anterior temporo-frontal regions (in all studied frequency bands) and in posterior regions in the $\gamma$ band. On the other hand, the connectivity pattern from healthy controls indicated two clusters of higher synchrotization. one among left temporal sensors and another one among central channels. Both of them were found in all frequency bands. In the $\gamma$ band. controls showed higher Synchronization Likelihood values than MCI patients between central-posterior and frontal-posterior channels and a high synchronization in posterior regions. The inter-hemispheric increased synchronization values could reflect a compensatory mechanism for the lack of efficiency of the memory networks in MCI patients. Therefore. these connectivity profiles support only partially the idea of $\mathrm{MCI}$ as a disconnection syndrome, as patients showed increased long distance inter-hemispheric connections but a decrease in antero-posterior functional connectivity.
\end{abstract}

Keywords: Disconnection syndrome, functional connectivity, magnetoencephalography, memory, mild cognitive impairment, synchronization likelihood

\footnotetext{
${ }^{1}$ These two authors have contributed equally to this work.

'Correspondence to: Ricardo Bajo, Laboratory of Cognitive and Computational Neuroscience Certer for Biomedical Technology
}

(CBT) Campus de Montegancedo 28660, Universidad Politécnica de Madrid, Spain. Tel.: +34 628909785; E-mail: ricbajo@gmail.coın. 


\section{INTRODUCTION}

The disruption of anatomic and functional connectivity in the brain of Alzheimes's disease (AD) patients due to neurofibrillary pathology [1] has led to the idea of conceptualizing the cognitive symptomatology as a disconnection syndrome [2]. This connectivity impairment suggests the existence of abnormal interactions between neutonal systems [3]. Because the pathophysiological characteristics of the disease seem to begin even decades before the cognitive symptomatology, it is of interest to evaluate whether functional connectivity profiles are affected in early clinical conditions such as Mild cognitive impaitment (MCI), as patients suffering from MCI present a higher risk of developing dementia (rate of conversion of $10-15 \%$ per year [4]). Whether the early anatomical connectivity impairment modulates the profiles of functional connectivity in MCI patients is still a matter of debate [5].

To describe how brain regions are coordinated to support higher cognitive functions, the tern functional comnectivity has been coined ([6,7], see [8], for a classification of different types of brain connectivity). Functional connectivity reflects the statistical interdependencies between two physiological signals, providing information about functional interactions between the corresponding brain regions. Long range synchronization between signals (oscillatory activity) originated in relatively distant neuronal populations, has been proposed as the mechanism for communication and integration of inforntation in the brain [9-11]. In fact, the binding phenontena in perception [12] or the forntation of new nuemories [13] seems to be based on synchronization, at specific frequency bands, between two oscillatory time series which reflect activity fron two brain regions.

Previous fMRI studies using functional connectivity in MCI patients have shown decreases [14-16] and increases in functional connectivity values $[15,16]$ in MCI patients as compared with healthy age-matched participants. However, although fMRI connectivity neasures provide spatially-resolved inforntation about connectivity patterns between brain regions, they do not directly reflect coupling between neuronal oscillators in different frequency bands known to play distinct roles in cognition (see [17] for an exantple on MCI). Fot this purpose various connectivity measures for magnetoencephalography (MEG)/electroencephalography (EEG) have been developed [18-20], as $M E G / E E G$ signals provide a direct measure of neuronal activity with high temporal resolution.
Here we use Synchronization Likelihood (SL) [21]. an index which provides a nonlinear characterization of functional connectivity, to: 1) describe the synchronization topologies that support memory success in MCI patients and healthy aging participants and thus evaluate the disconnection hypothesis and 2) evaluate whether these synchronization topologies allow to differentiate between MCI and age matched elderly controls. Moreover, the rationale for using SL in this study is two-fold: First, SL has been widely used as a functional connectivity nueasure in $\mathrm{AD}$ patients with both EEG [22,23] and MEG [24]. Second, it is a robust and nonlinear algorithm which overcomes the limnitations of linear approaches. SL could be complententary to other measures of functional connectivity such as mutual information and phase synchronization. More importantly, functional connectivity measures could add significant diagnostic information to other biomarkers such as anatomical connectivity or measures of amyloid- $\beta$ (A $\beta$ ) deposition and neurofibtillary tangles that probably represent the origin of anatomical disconnection in $\mathrm{AD}$. Disconnection in $\mathrm{AD}$ is associated with an increase in local symchronization [24]. The same finding in MCI would suggest that damage to anatomical conmectivity already occurs at this stage. On the other hand, an increase in long distance synchronization would be compatible with the use of alternative networks.

\section{MATERIALS AND METHODS}

\section{Participants}

Forty-one right handed, elderly patticipants recruited fron the Geriatric Unit of the Hospital Universitario San Carlos, Madrid, participated in the study. Participants were divided into two groups based on their clinical profiles: twenty-two participants were classified as multi-domain MCI patients, and the other nineteen as healthy control volunteers. Three MCI recordings were excluded from further analysis due to an excessive noise level.

MCI diagnosis was established according to the criteria proposed by Petersen ([4], see also [25]). To be diagnosed as having MCI, patients had to fulfill the following ctiteria: 1) report cognitive conplaints corroborated by an informant (a person who stays with the patient for half a day at least 4 days a week); 2) objective cognitive impairment, documented by delayed recall as measured by the Logical Memory II subtest of the Wechsler Memory Scale Revised (score below 17 
for 16 or more years of education; score below 9 for 8 to 15 years of education) and 1.5 Standard Deviations (SD) below ntean in test of executive functions such as WCST or Stroop; 3) normal general cognitive function, as determined by the clinician's judgment based on a structured interview with the patient and an informant: 4) a MMSE score greater than $24 ; 5$ ) relatively preserved daily living activities as measured by the Lawton scale; and 6) not sufficiently inıpaired, cognitively and functionally to meet criteria for dententia. As a result twenty-two participants were included in the MCI group. According to their clinical and neuropsychological profile, all participants in this group were considered nutti-dontain MCI patients [4]. Nineteen agematched, healthy elderly volunteers, without nnemory complaints, recruited for a project called "Aging with Health", at the San Carlos Hospital in Madrid consented to participate in the study. This group undergoes a complete medical revision every year. Patients and controls underwent a neuropsychological assessment, in order to establish their cognitive status in multiple cognitive functions. Specifically, ntemory impaitment was assessed by the Logical Mentory (imntediate and delayed) front the Wechsler Memory Scale-III-R. Two scales of cognitive and functional status were also applied: the Spanish version of the Mini Mental State Examination (MMSE) [26], and the Global Deterioration Scale/Functional Assessinent Staging GDS/FAST [27]. In order to avoid possible differences due to the years of education, patients and controls were chosen so that the resulting average nunber of years of education was sintilar: 10 years for patients and 11 years for controls. Table 1 summarizes the denographic and clinical information for both groups. The fact that out sample of MCI patients cante from a mentory clinic tather than from a population based sample could explain the high proportion of annestic multidomian MCI.

Before the MEG recording, all participants or legal representatives gave informed consent to participate in the study. The study was approved by the local ethics comnittee.

\section{Stimuli and task}

A modified version of the Stemberg's letter-probe task $([28,29])$ was used. A set of five letters was presented and the participants were asked to keep the letters in mind. After the presentation of the five-letter set, a series of single letters (1000 $\mathrm{ms}$ in duration with a random ISI between 2-3 s) was presented one at a time, and the participants were asked to press a button
Table 1

\begin{tabular}{lccccc}
\hline & Age & MMSE & GDS & LM1 & LM2 \\
\hline Cont'ol & $71.6 \pm 8$ & $29.5 \pm 0.7$ & 1 & $42.5 \pm 8^{*}$ & $26.7 \pm 7^{*}$ \\
MCI & $74.8 \pm 3$ & $27.7 \pm 1$ & 3 & $19.1 \pm 5$ & $13.1 \pm 6$ \\
\hline
\end{tabular}

MMSE, Mini-Mental State Examination: GDS, global deterioration scale; LM, logical memory.

${ }^{*} p<0.0001$ showing the differences in LM1 and LM2 between conisol and MCI cases.

with their right hand when a menber of the previous set was detected. The list consisted of 250 letters in which half were targets (previously presented letters), and the remaining letters were distracters (different from the previously presented letters). All participants completed a training session before the actual test, which did not start until the participant demonstrated that he/she could rentember the five-letter set. Letters were projected through a LCD video-projector (SONY VPL$\mathrm{X} 600 \mathrm{E}$ ), situated outside of the shielded-room onto a series of in-room mirrors, the last of which was suspended approxinately 1 meter above the participant's face. The letters subtended 1.8 and 3 degrees of horizontal and vertical visual angle respectively.

\section{MEG data collection}

The MEG signal was recorded with a $254 \mathrm{~Hz}$ sannpling frequency and a band pass of 0.5 to $50 \mathrm{~Hz}$, using a 148-channel whole-head magnetometer (MAGNES $2500 \mathrm{WH}, 4-\mathrm{D}$ Neuroimaging) confined in a magnetically shielded room. An environmental noise reduction algorithnt using reference channels at a distance from the MEG sensors was applied to the data. Thereafter, single trial epochs were visually inspected by an experienced investigator, and epochs containing visible blinks, eye movements or nuscular attifacts were excluded from further analysis. Artifact-free epochs from each channel were then classified into four different categoties, according to the subject's perforntance in the experiment: hits, false alarms, correct rejections, and omissions. Only hits were considered for further analysis because we were interested in evaluating the functional connectivity patterns which support recognition success. 35 epochs were used to calculate SL values. This lower bound was determined by the participant with least epochs. To have an equal number of epochs across participants, 35 epochs were randonly chosen from each of the other participants.

In the only previous SL-Inenıry study we are aware of [30], a 1 minute time-window was used for SL analysis. Such a long time-window makes it difficult to ensure the homogeneity of the cognitive processes in- 


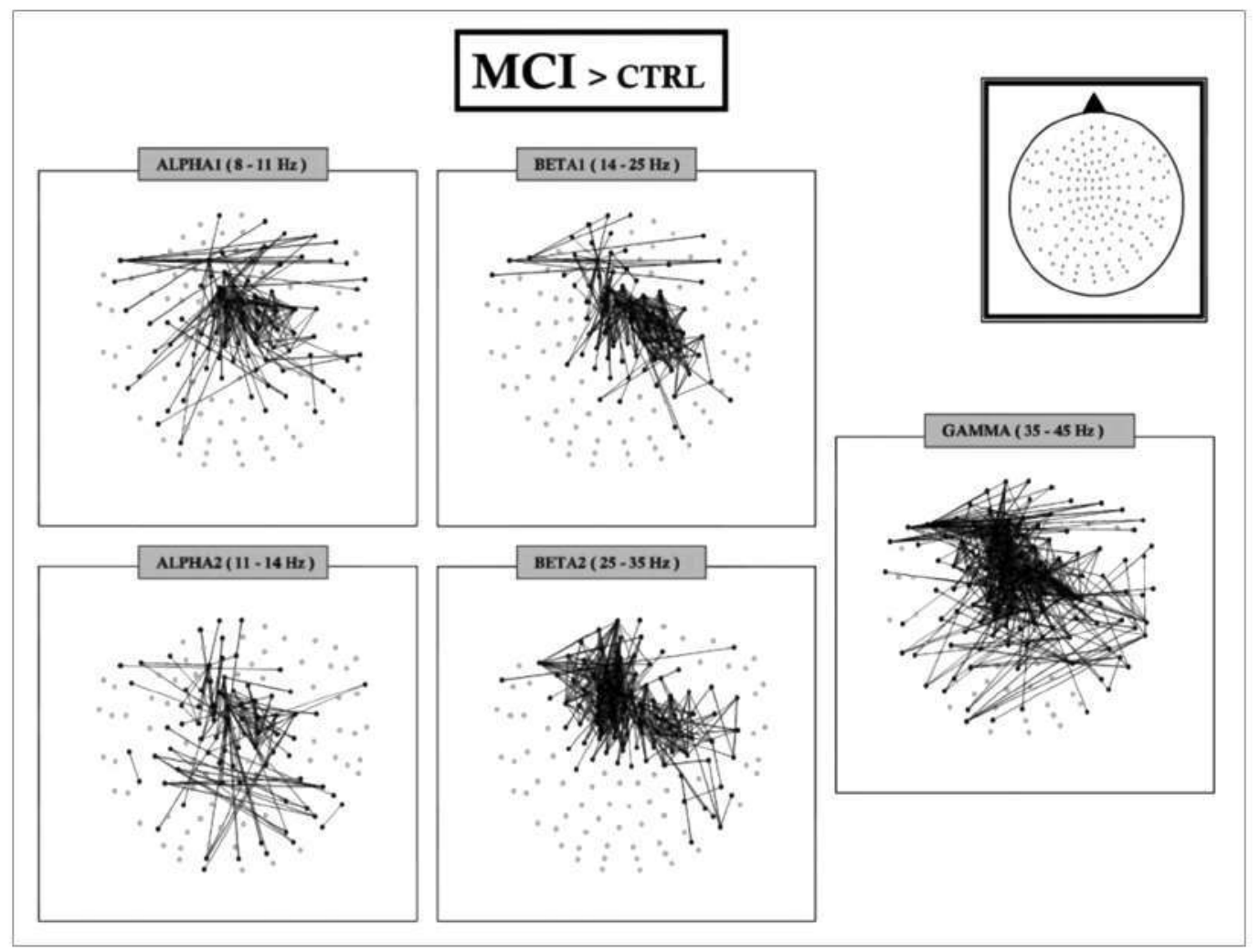

Fig. 1. Significant differences in SL between electrode pairs for different frequency bands. (MCI $>$ Control). False Discovery Rate type I was applied.

volved. Thus, it seems convenient to apply the traditional SL algorithm to a shorter time window. This will lead to an Event Related-SL (ER-SL) that achieves the stability of functional connectivity patterns across participants during the memory task.

In-house Fortran code was used to implement the SL algorithm as described by [21]. The SL algorithm was applied to the 35 extracted artifact-free one second epochs for each subject. For each frequency band optimal SL parameter values were chosen according to [31] for each frequency band and one second length:

Lag: $\mathrm{L}=f_{\mathrm{s}} /(3 * \mathrm{HF})$,

Embedding dimension: $\mathrm{M}=3 * \mathrm{HF} / \mathrm{LF}$,

Theiler window: $\mathrm{W} 1=2 * \mathrm{~L} *(\mathrm{M}-1)$, Pref below 0.05 ,

Window length: W2 > 10/Pref $+\mathrm{W} 1-1$.

Where $f_{s}$ sampling rate, and HF and LF are the high and low frequency bound, respectively.

The following frequency bands were considered: alpha1 $(\alpha 1,8-11 \mathrm{~Hz})$, alpha2 $(\alpha 2,11-14 \mathrm{~Hz})$, beta1 $(\beta 1,14-25 \mathrm{~Hz})$, beta2 $(\beta 2,25-35 \mathrm{~Hz})$, gamma $(\gamma$, $35-45 \mathrm{~Hz}$ ). The SL index was not computed for bands under $8(\mathrm{~Hz})$ as the epoch length and sampling rate do not allow an accurate enough estimation [31].

All epochs were digitally filtered off-line at the above frequency bands. Subsequently, the SL was calculated for each of the 35 one-second epochs with $148 * 147 / 2$ channel pairs for each frequency band, unfiltered epochs, and each subject (19 controls and 19 patients).

\section{Statistical analysis}

To compare the level of SL between the 2 groups, SL values were first averaged across epochs for each participant and channel pair. Then, False Discovery Rate [32, 33] was applied to find channel pairs with significant differences between groups. For each channel pair a between-groups Kruskal-Wallis (non-parametric) test was calculated. From the resulting p-values a significance threshold was calculated with a corresponding $q=0.2$ ( $q=0.4$ in $\alpha$ band) using the type I false discovery rate implementation from [33].

Additionally, we analyzed similarities in the connectivity patterns of MCI patients and controls at the in- 


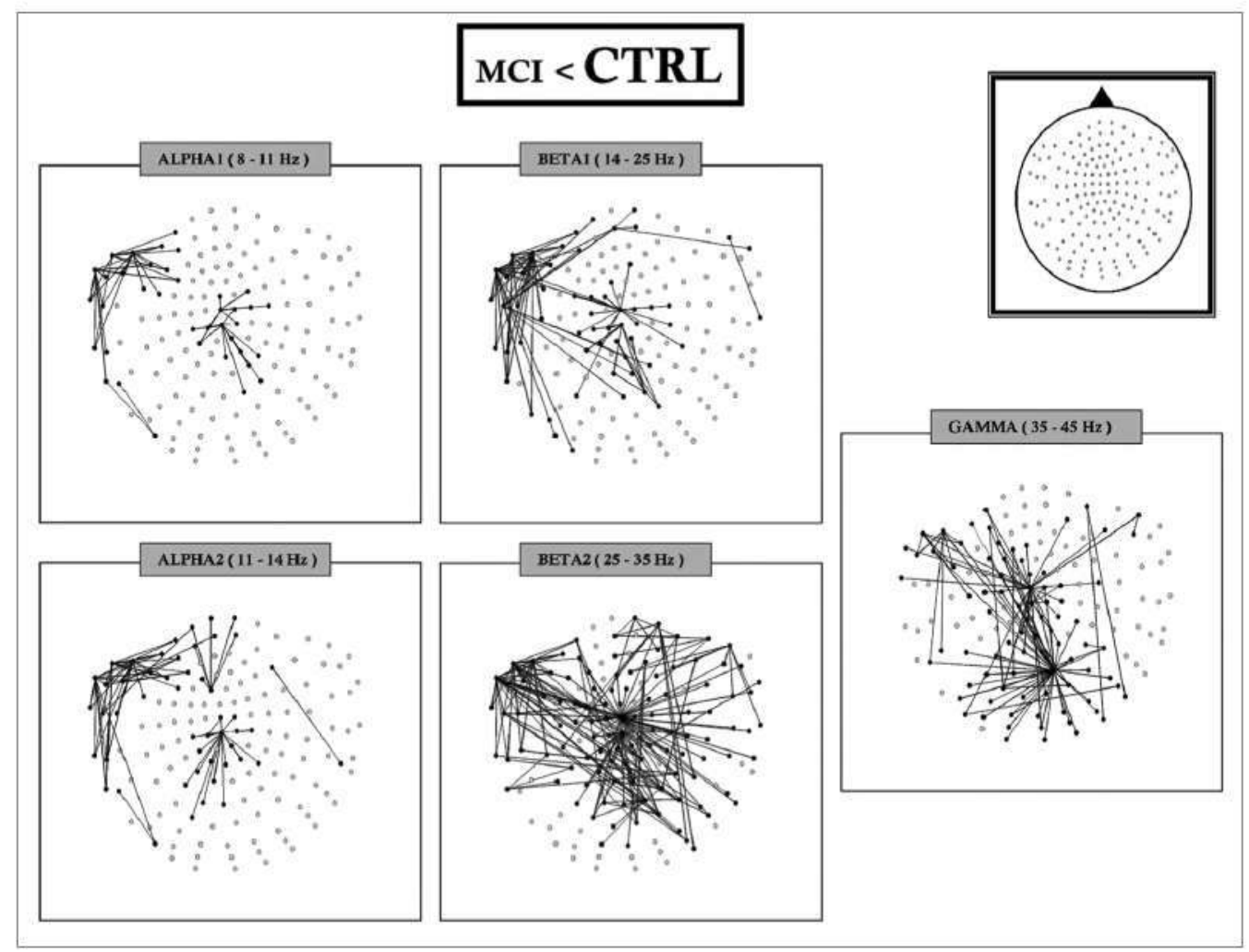

Fig. 2. Significant differences in SL between electrode pairs for different frequency bands. (MCI $<$ Control). False Discovery Rate type I was applied.

dividual level. For each individual we report connectivity values 2 standard deviations above or below the average value across all channel pairs.

\section{RESULTS}

\section{Behavioral performance}

Behavioral performance during the memory task revealed no significant differences between groups, either with respect to the targets (total number of hits, misses and reaction time), or to the distracters (correct rejections, false alarms and reaction time). The percentage of hits ( $80 \%$ control group and $84 \%$ MCI group) and correct rejections (92\% control group and $89 \% \mathrm{MCI}$ group) was high enough in both groups, indicating that participants actively engaged in the task.

\section{$M E G$ profiles of functional connectivity}

\section{$M C I>$ Control participants}

Comparing both groups (see Fig. 1), MCI patients showed a clear cluster of higher synchronization values over the anterior and central regions in all frequency bands. Additionally, MCI patients showed higher interhemispheric SL values than the control group between left and right temporo-frontal sensors in all frequency bands. Finally, only in $\gamma$ band, MCI subjects present a pattern of higher inter-hemispheric SL than controls in posterior regions.

\section{$M C I<$ Control participants}

Two non-functionally related clusters of local interactions showed higher SL values in the control group: one among left temporal sensors and another one in central-posterior channels. Both of them were found in all frequency bands. Additionally, controls showed higher SL values, in $\gamma$ and $\beta 2$ bands, between central and posterior channels and between frontal and posterior regions. Finally, also in $\gamma$ band, there is a higher posterior synchronization in controls when compared to patients (see Fig. 2).

Single-patient analysis: MCI and control participants

We calculated the average SL for each participant. SL values above and below two standard deviation from 


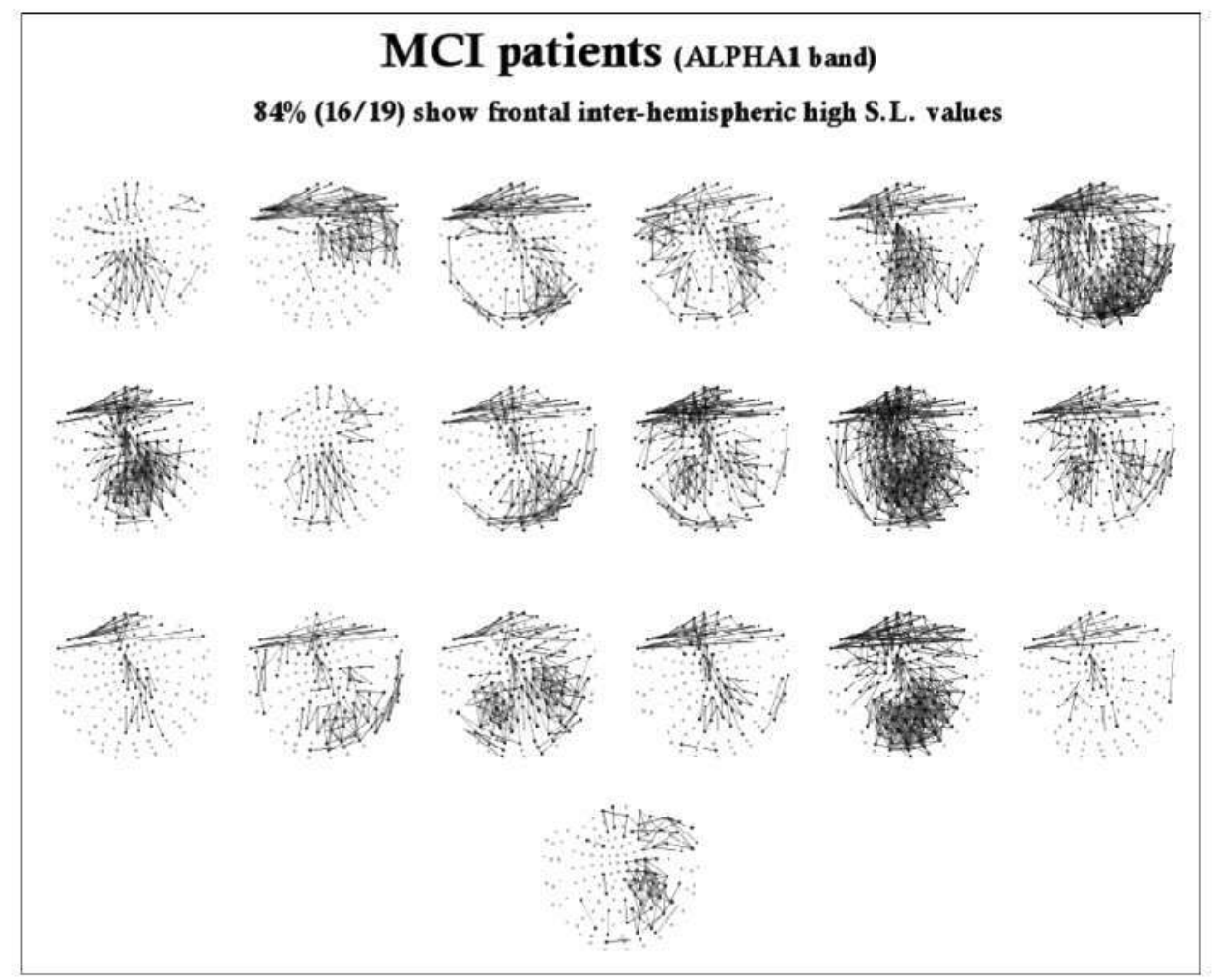

Fig. 3. SL for individual MCI patients $(N=19)$ in the $\alpha 1$ band. Black lines indicate electrode pairs with SL two standard deviations above or below the individual SL average. 16 out of 19 patients show high frontal interhemispheric SL values.

the average are shown (Fig. 3). Profiles of synchronization in Fig. 3 resemble those of the between-group comparison (Figs 1 and 2). Although results for the $\alpha 1$ band are shown they were similar for other frequency bands. MCI patients present a remarkable pattern of high inter-hemispheric SL values between left and right temporo-frontal sensors. In order to get a clearer figure, connections between nearest neighbours were not plotted. Most of the MCI patients (16/19) showed a high anterior inter-hemispheric SL in all frequency bands. In contrast, this pattern appears only in $7 / 19$ control participants.

\section{Receiver operating characteristic (ROC) curve}

To assess the ability of SL to discriminate between $\mathrm{MCI}$ and control participants during successful memory encoding, we calculated the receiver operating characteristic (ROC) curve [34,35], which provides a graphical representation of the trade-off between sensitivity and specificity as a function of the classification threshold. We took the average SL across channel pairs showing significant differences between controls and patients as a classification variable.
Figure 4 shows the ROC curve for the mean SL across channel pairs where control $>\mathrm{MCI}$, for $\beta$ and $\gamma$ frequency bands. High values of area under the ROC curve (between $72 \%$ and $82 \%$ ) were found, indicating that the connectivity patterns for the two groups were clearly different.

\section{DISCUSSION}

Our study is partly motivated by the question of whether MCI can be considered a disconnection syndrome. The results show a differential synchronization topography between MCI patients and controls. In general, while patients showed a tendency for higher interhemispheric synchronization among anterior sensors, controls showed a distinctive pattern with higher SL values over the left hemisphere and posterior regions, in addition to interconnections between fronto-posterior regions in $\beta 2$ and $\gamma$ bands. The fact that controls and patients used different memory networks suggests that MCI patients were compensating for the inefficiency of the antero-posterior connections, in agreement with previous findings [36]. 


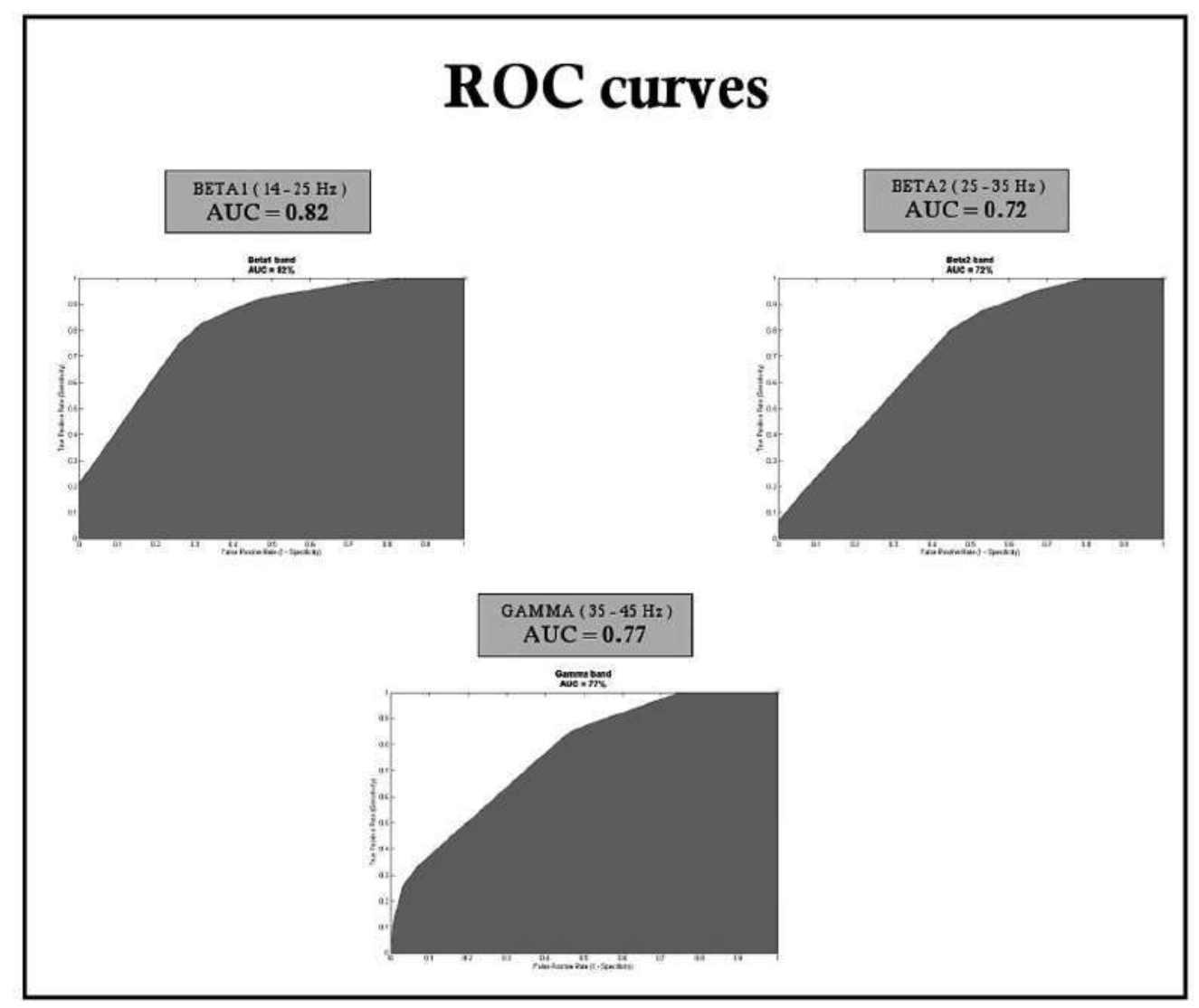

Fig. 4. Receiver Operating Characteristic for $\beta$ and $\gamma$ frequency bands. The variable used for classification was the average SL across sensor pairs with significantly higher values for controls as compared to patients. $\mathrm{AUC}=\mathrm{Area}$ under the curve.

The higher inter-hemispheric synchronization in MCI patients among anterior regions was present in all frequency bands considered. It is of interest that, as we can see in figure 3 , sixteen out of nineteen MCI patients showed anterior inter-hemispheric connectivity, which reflects the stability of the connectivity profiles across patients. Previous working memory EEG studies have shown increased inter-hemispheric coherence in MCI patients in the $\delta$ to $\beta$ range [37-39]. The synchronization cluster over the central regions, also present in the $\mathrm{MCI}$ group, has been previously described in $\mathrm{AD}$ patients, although the AD group showed a more posterior pattern than our MCI patients see [24]. The increase in synchronization in certain areas in patients may be the result of a loss of functional interactions in other regions. An example of increased synchronization by disconnection has been demonstrated by pharmacological isolation of one hemisphere by amobarbital injection, which induces an increase of $\beta$ band synchronization over the non-injected hemisphere [40]. Thus, an impairment of certain brain networks can induce an in- crease in synchronization in other networks, probably reflecting a compensation mechanism. A pattern of higher synchronization in MCI patients has been described previously in the only EEG study that calculated the SL during a memory task [30]. These authors found higher short-distance $\alpha$ band $(8-10 \mathrm{~Hz})$ SL values for the MCI group as compared to a group of elderly participants with subjective memory complaints. By contrast, in our study we found long distance synchronization in several frequency bands in comparison to a group of healthy controls without memory complaints. The fact that we were evaluating only hit responses and the use of a working memory instead of an episodic memory task could be a source of differences between studies.

In all frequency bands, controls showed higher SL values over the left hemisphere, particularly among left temporal sensors. The fact that participants were all right handed and were performing a verbal memory task, may explain the involvement of left temporal regions. It has been recently suggested that the functional 
coupling of $\alpha$ thythıns could constitute a neural substrate for language lateralization [41]. The increased synchronization in controls ntay reflect a more efficient processing of verbal stimuli.

Differences between groups in the $\gamma$ band were of great interest. In addition to the main differences found in the other bands, MCI patients showed an increase in posterior inter-hemispheric connectivity while controls showed long distance interconnection between frontal and posterior regions acconıpanied by a posterior synchronization cluster. Differences in this band have not been frequently reported in EEG studies (see [42] for a recent example; see [43] for a $\gamma$ study). This difference between EEG and MEG inight be ascribed to a better signal to noise ratio in high frequency bands in MEG as compared to EEG, as biological tissue is much more transparent to magnetic fields than to electrical potentials, making MEG more sensitive to $\gamma$ band effects. It has been proposed that the $\gamma$ band plays an important role in high cognitive processing such as in the integration of information [44] or in attention [45]. In fact, Kaiser et al. [46] propose that $\gamma$ band nodulations reflect the synchronization of sub-processes in neuronal networks related to cognition. Several studies have related $\gamma$ activity to memory processes [47]. Specifically, cross-frequency coupling between the $\gamma$ and $\theta$ bands in the medial temporal lobe is a predictor of memory performance $[48,49]$.

An interaction between frontal and parietal regions during working memory tasks can be expected, as revealed by lesions in anintals and humans and by functional neuroinaging studies [50]. Furthet more, functional dependencies between frontal and posterior areas have been previously associated with menory formation and recovery [49]. Thus, the pattern of long distance SL in the $\gamma$ band found in the control group could reflect a fronto-posterior interaction associated with the working memory task. Higher connectivity in healthy elders than $\mathrm{AD}$ patients between these areas has been previously reported [23]. In our study, MCI patients ntay rely less on the fronto-posterior connection to perform the task and nore on alternative networks. Thus, the pattern of increased inter-hentispheric connectivity in MCI Inay represent a transitory physiological state as MEG and EEG studies of functional connectivity in AD patients showed a decrease in $\gamma$ band synchronization $[43,51]$.

Since only hit responses were included in our analysis we cannot conclude that differences in synchronization profiles imply that patients use a less efficient network. In fact, $\alpha$ - and $\beta$-synchronization have been related to successful perfornance in shott-ternı nıemory tasks $[52,48]$. Thus, between groups profile differences of SL could indicate that control and patients were using a different cognitive strategy to perform the task.

In general, the increased inter-hemispheric synchronization in MCI patients could be interpreted as a compensatory mechanism. In fact, our results seem to agree with the Hemispheric Asynıntetry Reduction in Older adults (HAROLD) nodel. The HAROLD nodel states that, under sintilar circunsstances, prefrontal activity during cognitive perfotmance tends to be less lateralized in older than in younger adults [53]. Thus, according to this model, older adults rectuit right hentisphere frontal regions to compensate for the loss of efficiency of the left hemisphere networks. This compensatory mechanism found in aging seems to be $\mathrm{ex}$ haustively used by the MCI patients, suggesting that they recruit more often right hemisphere regions to perform the verbal task. Sintilarly, when the complexity of the task increases, young participants tend to recruit right hentisphere regions during the perfornance of verbal tasks [54]. Thus, it could be hypothesized that the increase in inter-henispheric synchronization in MCI patients reflects the recruitment of right hemisphere areas to compensate for: 1) the loss of efficiency of the left hemisphere verbal memory networks and 2) the reduced synchronization of the fronto-posterior networks.

Functional connectivity fMRI studies in MCI patients showed higher connectivity values within the posterior regions $[15,16]$. Here we show that this patteri of posterior interactions is extended to the anterior tegions of the brain. Differences between studies could be due to the type of analysis performed in each study (frequency domain versus blood flow) [55] and the type of responses considered in each of them as in the fMRI studies "hits" were not always considered separately from other types of responses.

We hypothesized that the increase of synchronization in MCI patients could be interpreted as a compensatory strategy for the loss of cognitive efficiency of the niemory networks that are norntally activated in healthy age-ntatched subjects. Previous functional neuroinaging and physiological studies have described other possible signs of this compensatory process such as the increase in activity in the medial temporal lobe [56] or the plasticity of the cholinergic system [57]. This finding opens the possibility to neuropsychological interventions benefitting from the existence of spared cortical networks related to memory success in MCI pa- 
tients. Additionally, the patterns of synchronization topography found in this study reveal a tendency to use differential menory networks in each group to achieve similar task performance. This is probably due to the use of different recognition strategies in each group.

It could be argued that the inter-hemispheric patterns of functional connectivity found in MCI patients are not compatible with the reported damage in white matter found in this group of patients [58]. Nevertheless, MCI patients show loss of axons but not dentyelination. Thus, while AD patients show note extensive damage to white natter [59], MCI patients denonstrate an anatonical connectivity that is better preserved, opening the possibility for long distance communication [60]. These findings to not argue against the notion of $\mathrm{AD}$ as a disconnection syndrome. Rather, our data suggest that during MCI the brain is still able to compensate for potential initial anatomical defects resulting in an increase of functional connectivity, before AD developinents.

In conclusion, the fact that MCI patients are able to establish long distance connections argues against the disconnection hypothesis in this neurological condition. This higher anterior interhentispheric synchronization profile showed by the MCI group could reflect the use of a different cognitive strategy to solve the task because: 1) controls used an anterior-posterior profile of connectivity and 2) profiles of connectivity were obtained for hit trials when processing has been efficient. Thus, the lack of observed disconnection syndrome is due to an apparent compensatory ntechanissn in MCI subjects. The existence of anterior interhentispheric synchronization could be related to the fact that our sanuple is fornted by amnestic-multidomain MCI. Since in the ntajotity of the cases ntenory dysfunction was accompanied by executive impairment, the deficit in frontal lobe cognitive abilities would lead to increased compensatory interhemispheric synchronization over this brain region. In fact, there is an increasing evidence of a compensatory response in MCI and early $\mathrm{AD}$ see [61] and there are certainly nodels related to this issue $[62,63]$.

The fact that our sample of MCI subjects was fornted essentially by amnestic multidomain MCI subjects (which is associated a more severe state than single donain anınestic MCI) could explain the more prominent difference between MCI subjects and controls. Future studies should evaluate the ability of MEG and SL to differentiate between single domain amnestic MCI subjects and controls. Moreover, the fact that there were clear differences between groups indicates that
MEG derived connectivity measures could contribute to the diagnosis of MCI. Future studies should evaluate whether this SL profiles are useful in predicting which of the MCI patients will develop dementia.

\section{ACKNOWLEDGMENTS}

This study was partially funded by the Madr.IB (CAM i+d+I project) and the Spanish Ministry of Science (SEJ-2006-07560).

RG is financially supported by the Spanish Ministry of Science and Innovation (FPI fellowship SEJ2006$07560)^{\prime}$.

AN was supported by a Ramón y Cajal research contract (Spanish Ministry of Science and Innovation/UCM).

Authors' disclosures available online (http://www.jalz.cont/disclosures/view.php?id=489).

One of the authors (M. Sancho) acknowledges support fron the Universidad Complutense de Madrid under Project No. 910305 for Bioelectromagnetics Research Group.

\section{REFERENCES}

[1] Braak H, Braak E (1991) Newropathological stageing of Alzheimer-related clianges. Acta Netropathol 82, 239-259.

[2] Morrison JH, Scherr S, Lewis DA, Campbell MJ, Blooin FE, Rogers J, Benoit R (1986) The laininar and regional distribution of neocortical somatostatin and neuritic plaques: Implications for Alzheimer's disease as a global neocortical disconnection syndrome. The Biological Substrates of Alzheimer's Disease, Academic Press, Orlando, pp. 115-131.

[3] Delbeuck X, Van der Linden M, Collette F (2003) Alzheimer's disease as a disconnection symdrone? Neuropsychol Rev 13 , 79-92.

[4] Petersen RC (2004) Mild cognitive impaimment as a diagnostic entity. J Intern Med 256, 183-194.

[5] Stam CJ, de Haan W, Dafferishofer A, Jones BF, Manshanden I, van Cappellen van Walsun AM, Montez T, Verbunt JP, de Munck JC, van Dijk BW, Bejendse HW, Scheltens P (2009) Graph theoretical analysis of magnetoencephalographic functional connectivity in Alzheimer's disease. Brain 132, 213-224.

[6] Friston KJ (1994) Functional and effective connectivity in neuroimaging: a synthesis. Human Brain Mapp 2, 56-78.

[7] Friston KJ (2001) Brain function, nonlinear coupling, and neuronal transients. Netrosctentist 7, 406-418.

[8] Sporns O, Chialvo DR, Kaiser M, Hilgetag CC (2004) Organization, developmem and function of complex brain networks. Trends Cogn ScI 8, 418-425.

[9] Varela F, Lachaux JP, Rodriguez E, Martinerie J (2001) The brainweb: phase synchronization and large-scale integgation. Nat Rev Neurosci 2, 229-239. 
[10] Fries P (2005) A mechanism for cognitive dynamics: neuronal comınunication through neuronal coherence. Trends Cogn $S_{C i}$ 9, 474-480.

[11] Engel AK, Fries P, Singer W (2001) Dynamic predictions: oscillations and synchrony in top-down processing. Nat $R e v$ Neurosci 2, 704-716.

[12] Singer W (1999) Neuronal synchrony: a versatile code for the definition of relations? Netron 24. 49-25.

[13] Fernández G, Effern A, Gruirwald T, Pezer N, Lehnertz K, Düınpelınann M, Van Roost D, Elger CE (1999) Real-tiıne tracking of memory formation in the human shinal cortex and hippocanpus. Sclence 285, 1582-1585.

[14] Grady CL, McIntosh AR, Beig S, Keigltuley ML, Burian H, Black SE (2003) Evidence from functional netroimaging of a compensatory prefrontal network in Alzheimer's disease. $J$ Neurosci 23, 986-993.

[15] Bokde AL, Lopez-Bayo P, Meindl T, Pechler S, Born C, FalIraco F: Teipel SJ, Moller HJ, Hampel H (2006) Functional comectivity of the fusiform gyrus du'ing a face-matching task in subjects with onild cognitive impairment. Brain 129, 1113-1124.

[16] Bai F, Zhang Z, Yu $H$, Shi Y, Yuan $Y$, Zhu W, Zhang $X$, Qian Y (2008) Default-mode network activity distinguishes annestic type inild cognitive impairmein from healthy aging: a combined structural and resting-state functional MRI study. Netroscl Lett 438, 111-115.

[17] Osipova D, Ranianen K, Ahveninen J, Ylikoski R, Happola $O$, Sirandberg T, Pekkonen E (2006) Source estimation of spontaneous MEG oscillations in mild cognitive impairment. Neurosei Lett 405, 57-61

[18] David O, Cosinelli D, Friston KJ (2004) Evaluation of different measures of functional connectivity using a neural mass model. Netrolmage 21.659-673.

[19] Pereda E, Quiroga RQ, Bhattacharya J (2005) Nonlinear Inultivariate analysis of neurophysiological signals. Prog Neuroblol 77, 1-37.

[20] Ausari-Asl K, Senhadji L, Bellanger JJ, Wendling F (2006). Quantilative evaluation of linear and nonlinear methods characterizing interdependencies between brain signals. Phys Rev E Stat Noniln Sof Matter Pbys 74, 031916

[21] Stain CJ, Dijk BW van (2002) Synchronization likelihood: an unbiased measure of generalized synchronization in multivariate data sets. Physica $D$ 163, 236-241

[22] Stam CJ. Montez T, Jones BF, Rombouts SA, van der Made Y, Pijnenburg YA, Scheltens P (2005) Disturbed fluctuations of resting state E.E. synchronization in Alzheimer's disease. Clin Netrophysiolo 116, 708-715.

[23] Babiloni C, Ferri R, Binetti G, Cassarino A, Dal FG, Ercolani M, Ferreri F, Frisoni GB, Lanuzza B, Miniussi C, Nobili F, Rodriguez G, Rundo F, Stan CJ, Musha T, Vecclio F, Rossini PM (2006) Fronto-pan'jetal coupling of brain thythms in mild cognitive impairment: a multicemtric EEG study. Brain Res Bull69, 63-73.

[24] Stam CJ, Jones BF, Manshanden I, van Cappellen van Walsum AM, Montez T, Verbunt JPA. (2006) Magnetoencephalographic evaluation of resting-siate functional connectivity in Alzheimer's disease. Neuroimage 32, 1335-44

[25] Gu'undman M. et al. (2004) Mild cognitive impaimment can be distinguished from Alzheimer disease and normal aging for clinical trials. Arch Neurol 61, 59-66.

[26] Lobo A, Ezquena J, Gomez BF, Sala JM, Seva DA (1979) [Cognocitive mini-test (a simple practical test to detect intellectual changes in inerdical patients)]. Actas Luso Esp Newol Psiquiatr Crenc Afines 7, 189-202.
[27] Auer S, Reisberg B (1997) The GDS/FAST staging system. Int Psychogeriatr 9(Supplemem 1), 167-171.

[28] deToledo-Morrell L, Evers S, Hoeppner TJ, Morrell F, Garron DC, Fox JH (1991) A 'stress' test for memory dysfunction. Electrophysiologic manifestations of eanly Alzheimer's disease. Arch Neurol 48, 605-609.

[29] Maestu F, Fernandez A, Simos PG, Gil-Gregorio P, Amo C, Rodriguez R, Arrazola J, Ortiz T (2001) Spatio-temporal patterns of brain magnetic activity during a memory task in Alzheimer's disease. Nemoreport 12, 3917-3922.

[30] Pijnenluw'g YA, Made Y, van Cappellen van Walsum AM, Knol DL, Scheltens P, Stano CJ (2004) EEG synclironization likelihood in mild cognitive impairment and Alzheines's disease during a working memory task. Chin Neurophysiol 115 , $1332-1339$

[31] Momez T, Linkenkaer-Hansen $K$, van Dijk BW, Stan CJ (2006) Synchronization likelihood with explicit timefrequency priors. Neuroimage 33, 1117-1125.

[32] Benjanini Y, Yekutieli D (2001) The control of the false discovery rate in inultiple testing under dependency. Am Stat $29(4), 1165-1188$.

[33] Genovese R, Lazan NA, Nichols T (2002) Thresholding of statistical maps in functional newoimaging using the false discovery rate. Neuroimage 15, 870-878.

[34] Metz CE (1978) Basic principles of ROC analysis. Semin NucI Med 8. 283-298.

[35] Hanley JA, McNeil BJ (1982) The meaning and use of the area under a recejver operating characteristic. (ROC) curve. Radiology 143, 29-36.

[36] Babiloni C, Feri' R, Bineni G, Vechio F, Frisoni GB, Lanuzza B, Miniussi C, Nobili F, Rodriguez G, Rundo F, Cassarino A, Infan'inato F, Cassetta E, Salina'i S, Eusebi F, Rossini PM (2009) Directionality of EEG synchronization in Alzheimer's disease subjects. Neurobiol Aging 30, 93-102

[37] Jiang ZY (2005) Abnorinal cortical functional connections in Alzheimer's disease: analysis of inter- and intra-hemispheric EEG coherence. J Zhejiang Unv Sci B 6, 259-264.

[38] Jiang ZY, Zheng LL (2006) Inter- and inira-hemispheric. EEG coherence in patients with mild cognitive impairment at rest and during working memory lask. J Zhejiang Univ $S_{c i} B$ ?, $357-364$.

[39] Jiang 2], Richardson JS, Yu PH (2008) The coniribution of cerebral vascular semicarbazide-sensitive amine oxidase to cerebral anyloid angiopathy in Alzheimer's disease. Neuropathol Appl Newrobiol 34, 194-204.

[40] Douw L, Baayen JC, Klein M, Velis D, Alphetis WC, Bot J, Heimans JJ, Reijneveld JC, Stam CJ (2009) Functional connectivity in the brain before and during inira-arterial amobarbital injection (Wada test). Neuroimage 46, 584-588.

[41] Brancucci A, Penna SD, Babiloni C, Vechio F, Capotosto P. Rossi D, Franciotti R, Torquati K, Pizzella V, Rossini PM, Romani GL (2008). Neuromagnetic functional coupling during dichotic listening of speech sounds. Hum Brain Mapp 29 . 253-64.

[42] van Deursen JA, Vuurunan EF, Verhey FR, van KranenMastenbroek VH, Riedel WJ (2008) Increased EEG gaınına band activity in Alzheimer's disease and mild cognitive inpaiment. INetral Transm 115, 1301-1311.

[43] Koenig T, Prichep L, Dierks T, Hub] D, Wahlund LO, John ER, Jelic $V$ (2005) Decreased EEG synclmonization in Alzheinner's disease and mild cognitive impairment. Neturobiol Aging 26, 165-171.

[44] Tallon-Baudry C, Berirand O (1999) Oscillatory gannı activity in humans and its role in object refoesentation. Trends 
Cogn ScI 3, 151-162

[45] Landau AN, Esterman M, Roberison LC, Bentin S, Prinzınetal W (2007) Differem effecis of voluniary and involuitary attention on EEG activity in the ganma band. I Neurosci 27, 11986-11990.

[46] Kaiser J, Lutzenberger W (2005) Human gatnuna-band activity: a window to cognitive processing. Neuroreport 16, 207 211.

[47] Buzsáki G (2006) Rhythms of the Brain. Oxford University Press.

[48] Fell J, Klaver P, Elfadil H, Schaller C, Elger CE, Fernandez G (2003) Rhinal-lippocampal theta colerence du'ing declarative memory formation: interaction with gainına synclmonization? Eur INeurosci 17, 1082-1088.

[49] Fernandez G, Tendolkar I (2001) Integrated brain activity in medial temporal and prefronial areas predicts subsequem memory performance: human declarative memory formation at the system level. Brain Res Bull 55, 1-9.

[50] Collette F. Hogge M. Salmon E, Van der Linden M (2006) Exploration of the neural substrates of executive functioning by functional neuroimaging. Neuroscience 139, 209-221.

[51] Stan CJ, van Cappellen van Walsum AM, Pijnenburg YA, Berendse HW, de Munck JC, Scheltens P, van Dijk BW (2002) Generalized synclironization of MEG recordings in Alzheimer's Disease: eridence for imolvement of the gamma band. J Clin Neurophyslot 19, 562-574.

[52] Klimesch W (1996) Menory processes, brain oscillations and EEG synchronization. Int I Psychophysiol 24, 61-100.

[53] Cabeza R, Dolcos F, Graham R, Nyberg L (2002) Similarities and differences in the newal correlates of episodic memory retrieval and working menory. Neuroimage 16, 317-330.

[54] Ryptna B, D'Esposito M (1999) The roles of prefrontal brain regions in components of working memory: elfects of memory load and individual differences. Proc NatI Acad Sci US A 96, 6558-6563.

[55] Sirotin YB, Das A (2009) Anticipatory hæemodynamic sig- nals in sensory contex not predicted by local neuronal activity. Nature 457, 475-479.

56] Dickerson BC, Salat DH, Greve DN, Chua EF, RandGiovannetti E, Rentz DM, Bertran L, Mullin K, Tanzi RE, Blacker D, Albert MS, Sperling RA (2005) Increased lippocampal activation in mild cognitive impairınent compared to normal aging and AD. Neurology 65, 404-411.

57] DeKosky ST, Ikonomovic MD. Styren SD, Beckett L. Wisniewski S, Bennett DA, Cochran EJ, Kordower JH, Mufson EJ (2002) Upregulation of choline acetyltransferase activity in hippocampus and frontal cortex of elderly subjects with mild cognitive impairment. Ann Neutrol 51, 145-155.

[58] Medina D, deToledo-Morrell L, Urresia F, Gabrieli JD, Moseley M, Fleischman D, Bennett DA, Leurgans S, Turner DA, Stebbins GT (2006) White matter changes in mild cognitive impairment and $\mathrm{AD}$ : A diffusion tensor inaging study. Neurobjol Aging 27, 663-672.

[59] Huang J. Friedland RP, Auclus AP (2007) Diffusion tensor imaging of normal-appearing white matter in mild cognitive impairment and early Alzheimes disease: preliminary evidence of axonal degeneration in the temporal lobe. $A J N R$ Am JNeuroradiol 28, 1943-1948.

[60] Ikonomovic MD, Abrahamson EE, Isanski BA, Wuu J, Mufson EJ, DeKosky ST (2007) Superior fromtal cortex cholinergic axon density in mild cognitive impaiment and early Alzheimer disease. Atch Neutrol 64, 1312-1317.

[61] Dai W, Lopez OL, Carmichael OT, Becker J, Kuller L, Gach $M$ (2009) Mild cognitive impairment and Alzheimer disease: patterns of altered cerebral blood flow at MR imaging. Radiology March 250, 856-866

[62] Satz P (1993) Brain reserve capacity on symptom onset after brain injury: A formulation and seview of evidence for threshold theory. Neuropsychology 7, 273-295.

[63] Stern Y (2009) Cognitive reserve. Neuropsychologia 47, 20152028. 